\title{
Stretchable Strain Sensors Fabricated by Screen Printing of Silver Paste on the Surface Modified Transparent Elastomeric Polyurethane Films
}

\author{
Chang Gyu Lee1, Bo Seok Kwon', Hyun Min Nam¹, Duck Min Seo², Jinwoo Park³, \\ Hyuc Hwangbo ${ }^{4}$, Lee Soon Park², Su Yong Nam ${ }^{1 *}$ \\ ${ }^{1}$ Department of Graphic Arts Information Engineering, Pukyong Natioinal University, Busan, Korea \\ ${ }^{2}$ School of Materials Science and Engineering, Ulsan National Institute of Science and Technology (UNIST), Ulsan, Korea \\ ${ }^{3}$ Screen Finetech Solutions Co., Ltd., Kyoto, Japan \\ ${ }^{4}$ FP Co., Ltd., Busan, Korea \\ Email: *suynam@pknu.ac.kr
}

How to cite this paper: Lee, C.G., Kwon, B.S., Nam, H.M., Seo, D.M., Park, J., Hwangbo, H., Park, L.S. and Nam, S.Y. (2018) Stretchable Strain Sensors Fabricated by Screen Printing of Silver Paste on the Surface Modified Transparent Elastomeric Polyurethane Films. Materials Sciences and Applications, 9, 1008-1020. https://doi.org/10.4236/msa.2018.913073

Received: November 13, 2018 Accepted: December 11, 2018 Published: December 14, 2018

Copyright (ㅇ 2018 by authors and Scientific Research Publishing Inc. This work is licensed under the Creative Commons Attribution International License (CC BY 4.0).

http://creativecommons.org/licenses/by/4.0/

\begin{abstract}
Strain sensors for human-motion detection must offer high stretchability, high sensitivity, fast response, and high recovery speed. In this study, we choose silver paste as a sensing material and use a screen printing method to fabricate the strain sensor based upon an electrical-resistance mechanism. After curing elastomeric polyurethane film with a thickness of $150 \mu \mathrm{m}$ on PET film, the polyester resin mixed with blocked isocyanate curing agent was coated as a masking layer to reduce the film's stickiness. The effect of the polyester masking layer upon the silver paste screen printing process was examined using a rolling-ball-tack test, TGA analysis of polyester resins, and cured silver-electrode films. The cost-effective strain sensor fabricated by using silver paste and screen printing processes on the stretchable-polyurethane-substrate film showed high sensitivity and fast response in a strain range of up to $100 \%$.
\end{abstract}

\section{Keywords}

Stretchable Strain Sensor, Screen Printing, Silver Paste

\section{Introduction}

Recent developments of new materials, fabrication processes, and sensing systems have contributed significantly to the achievement of thin, light-weight, flexible, and stretchable physical sensors. These offer a novel opportunity for 
human-activity monitoring and personal healthcare [1]-[7]. Flexible and stretchable physical sensors are capable of measuring human activities by sensing pressure, strain, and temperature. Pressure and strain sensors have similar structures and are based upon piezoelectricity-, piezoresistivity-, and capacitance-detection mechanisms. The pressure range can be divided into low $(<10$ $\mathrm{kPa})$, medium $(<100 \mathrm{kPa})$ and high $(>100 \mathrm{kPa})$ [3] subranges, while the strain regime can be classified into two categories of small-scale motions (e.g., subtle movement of the face, chest, and neck during emotional expression, breathing, swallowing, and speaking), and large-scale motion (e.g., bending movement of the hands, arms, and legs). The detection of these motions is of great importance for the application of strain sensors to monitoring and diagnosis of the human body for the purposes of good healthcare. Strain sensors for human-motion detection need to satisfy the requirements for high stretchability, sensitivity, fast response/recovery speeds, and durability/conformability.

Stretchable piezoelectric strain sensors have been fabricated using materials to convert mechanical energy into electrical signals. These strain sensors have high sensitivity, fast response and low power consumption. The sensing materials used include P (VDF-TrFE) [8] CNT composite [9] and ZnO NWs [10] [11] [12]. Sun et al. [13] fabricated an active-matrix strain sensor based upon a graphene transistor. The sensor had a piezopotential nano-generator and a coplanar-gate graphene transistor. Zhou et al. [14] designed a flexible strain sensor based on $\mathrm{ZnO}$ nanowire, which exhibited sensitivity with a gage factor up to 1250, high stability, and a fast response time. Few stretchable strain sensors using capacitive mechanisms have been reported, and these have used active materials such as SWCNTs/Ecoflex/SWCNTs [3], CNTs/PDMS/CNTs [4], AgNWs/Ecoflex/AgNWs [15], and SWCNTs/Silicone/SWCNTs [5].

The stretchable sensors mentioned above were fabricated using expensive piezoelectric and piezoresitive nano-materials combined with sophisticated electronic devices. To achieve stretchability, elastomeric materials such as polydimethyl siloxane (PDMS), polyurethane (PU), and commercial PU (Ecoflex) have been used; however, careful modifications of the stretchable substrates have not been studied in detail. In this study, we choose a cost-effective silver paste as a sensing material and use a screen printing method to fabricate the strain sensor based upon an electrical-resistance mechanism. A polyester resin mixed with blocked isocyanate curing agent was coated onto the commercial PU (Clear Flex 30) film to reduce the high tackiness of elastomeric PU, allowing the screen printing process to be applied to the formation of a strain sensor using a silver-powder/resin-composite layer as an active material.

\section{Experimental Methods}

\subsection{Fabrication of a Stretchable Film Substrate}

Clear Flex 30 polyurethane (CF PU) resins A and B (Smooth-on Inc., U.S.A) were mixed in a 50:50 wt\% ratio and coated onto polyethyleneterephthalate 
(PET) film with a release layer using a bar coater. The CF PU elastomeric coating on the PET film was allowed to level for $30 \mathrm{~min}$ at room temperature, followed by curing in a convection oven at $80^{\circ} \mathrm{C}$ for 1 hour and at $120^{\circ} \mathrm{C}$ for 2 hours. After curing the elastomeric PU film with a thickness of $150 \mu \mathrm{m}$ onto the PET film, the polyester resin (SK Chemical, Korea) mixed with the blocked isocyanate curing agent AA6627 (EO Nanochem, Korea) was coated and, then, thermally cured at $100^{\circ} \mathrm{C}$ for 1 hour and at $120^{\circ} \mathrm{C}$ for 2 hours to yield a thin film of thickness $3 \mu \mathrm{m}$. Figure 1 shows the coating machine and the multilayer structure of the stretchable substrate film for fabricating the strain sensor. Table 1 lists the physical data concerning the polyester resins, which were used to coat a masking layer of thickness $3 \mu \mathrm{m}$ on top of the elastomeric but sticky CF PU resin films.

\subsection{Preparation of Silver Paste}

Table 2 shows the formulation of the silver paste for making the resistive strain sensor on the stretchable PU film. Polyester resin (ES-215) with a molecular weight of $35,000 \mathrm{~g} / \mathrm{mol}$, glass-transition temperature $(\mathrm{Tg})$ of $-11^{\circ} \mathrm{C}$, and softening temperature of $100^{\circ} \mathrm{C}$ was used as the binder polymer of the silver paste. The solvent 2-(2-ethoxyethoxy)ethyl acetate (ECA) was mixed with the polyester resin at 50:50 wt $\%$ and, then, was stirred at $70^{\circ} \mathrm{C}$ for 24 hours to create the binder polymer solution, followed by filtration with 400-mesh filter cloth.

Three different silver particles were used to make silver paste in the polyester solution; Ag-1 powder (flake; length $=1-3 \mu \mathrm{m}$, thickness $\mathrm{d}=50 \mathrm{~nm}$ ), Ag-2 powder (flake; length $=1-3 \mu \mathrm{m}$, thickness $\mathrm{d}=100 \mathrm{~nm}$ ), and Ag-3 powder (flake; length $=7 \mu \mathrm{m}$, thickness $\mathrm{d}=1.96 \mu \mathrm{m}$ ). Figure 2 shows SEM images of the Ag-1, -2, and -3 powders. The weight ratio of Ag-1:Ag-2:Ag-3 was 15:15:40 (accounting for a total of $70 \mathrm{wt} \%$ of the silver paste), and the weight ratio of

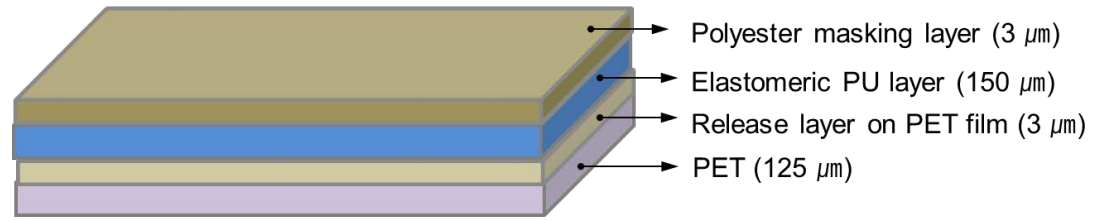

(a)

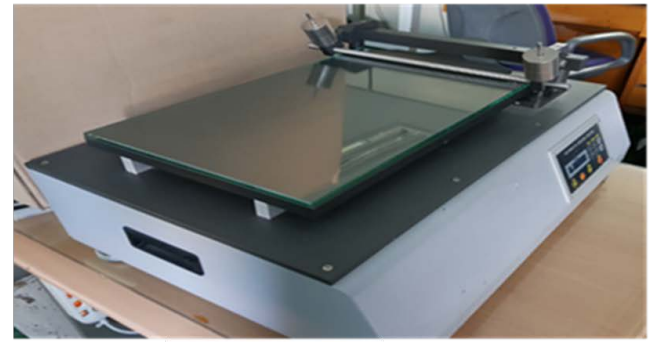

(b)

Figure 1. (a) Multilayer structure of elastomeric PU film substrate and (b) coating machine for fabrication of a stretchable strain sensor. 
Table 1. Physical data concerning polyester resins used to coat a masking layer onto the elastomeric PU substrate film.

\begin{tabular}{ccccc}
\hline Polyester resin & $\begin{array}{c}\mathrm{Tg} \\
\left({ }^{\circ} \mathrm{C}\right)\end{array}$ & $\begin{array}{c}\mathrm{OH} \\
\text { value }\end{array}$ & $\begin{array}{c}\text { Molecular weight } \\
(\mathrm{Mw}: \mathrm{g} / \mathrm{mol})\end{array}$ & $\begin{array}{c}\text { Softening temperature } \\
\left({ }^{\circ} \mathrm{C}\right)\end{array}$ \\
\hline ES-365 & 16 & $2-6$ & 40,000 & 120 \\
ES-215 & -11 & $2-6$ & 35,000 & 100 \\
ES-500 & 10 & $2-6$ & 26,000 & 90 \\
ES-360M & 17 & $7-11$ & 32,000 & 110 \\
\hline
\end{tabular}

Table 2. Formulation of silver paste.

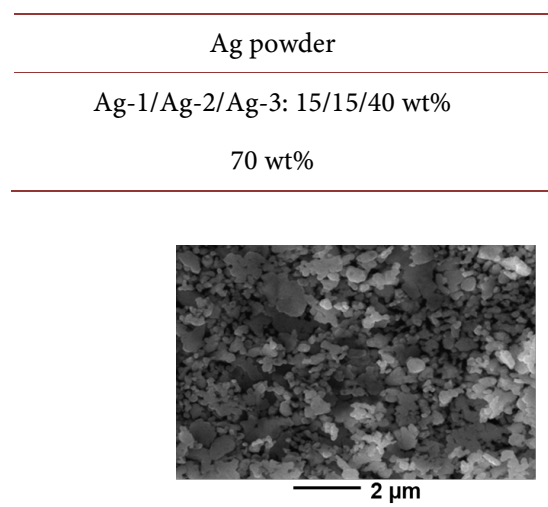

(a) $\mathrm{Ag}-1$

\begin{tabular}{ccc} 
Binder & Solvent & Additive \\
Polyester resin & ECA & BYK-180 \\
$10 \mathrm{wt} \%$ & $19 \mathrm{wt} \%$ & $1 \mathrm{wt} \%$ \\
\hline
\end{tabular}

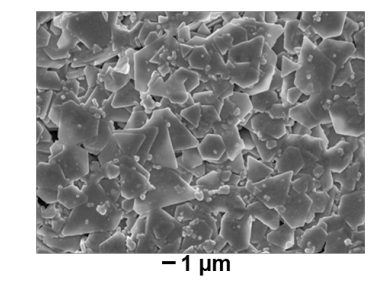

(c) $\mathrm{Ag}-3$

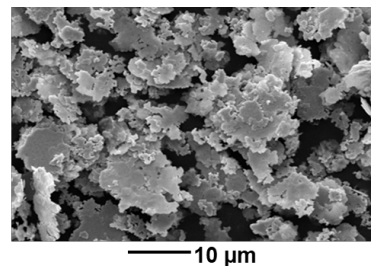

(b) $\mathrm{Ag}-2$

Figure 2. The SEM images of the Ag-1, -2 , and -3 powders.

polyester resin: ECA solvent: BYK-180 (BYK Co., Germany) dispersant was 10:19:1 wt $\%$, comprising the rest of the silver paste. Figure 3 shows the procedure for making silver paste. The silver-powder was poured into the polyester solution, followed by the addition of BYK-180 dispersant; then, the mixture was rotated at room temperature for 2 hours in the vacuum-defoaming mixer.

The electrode pattern shown in Figure 4 was made using a 250-mesh screen plate. After printing the silver paste onto the polyester masking layer/CF PU-substrate film, the electrode pattern was cured at $130^{\circ} \mathrm{C}$ for $30 \mathrm{~min}$ in a convection oven. To monitor the resistance, change of electrode pattern upon stretching, two copper-wire leads were connected securely to the electrode pattern using a silver-epoxy paste.

\subsection{Viscosity Measurement and Tackiness Test}

The silver paste was further mixed using a 3-roll mill at room temperature for 1 


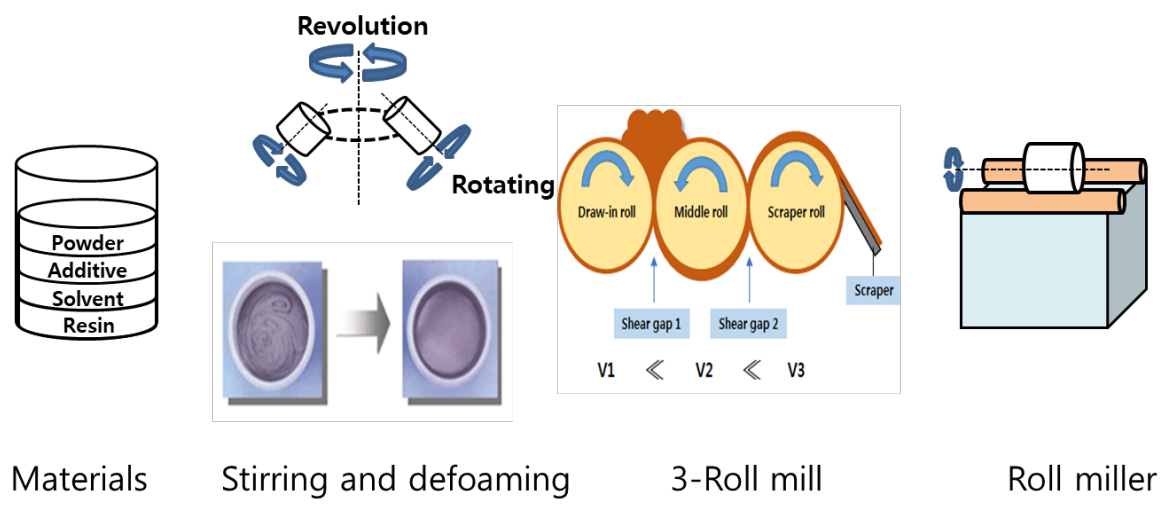

Figure 3. Silver paste-preparation procedure.

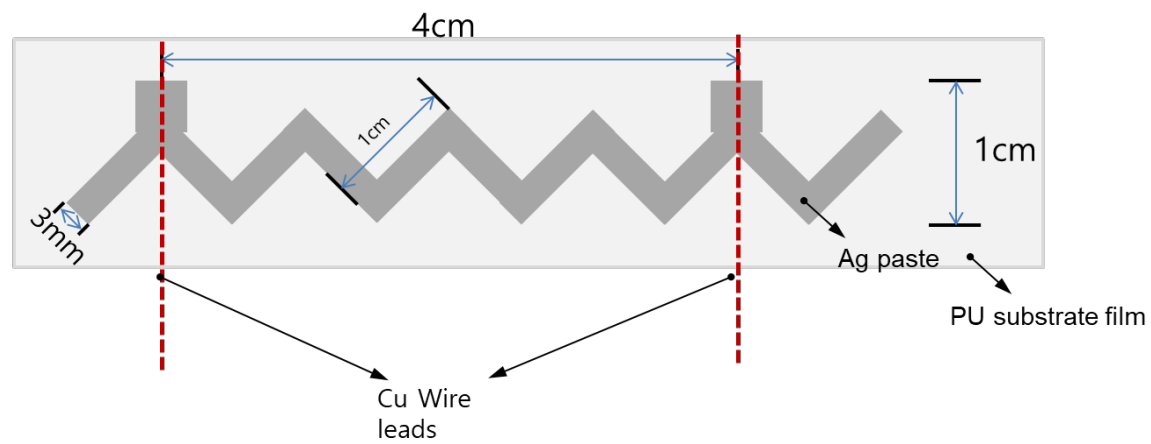

Figure 4. Silver-electrode pattern for screen printing on the polyester masking layer/PU-substrate film.

hour and, then, was stored in the $0^{\circ} \mathrm{C}$ chiller. Then, it was rotated on the roll miller for 2 hours at room temperature before being used for screen printing. The rheological properties of the silver paste were measured with thermos HAAKE Rheowin Pro 2.92 (Germany) at $23^{\circ} \mathrm{C}$.

The masking layer was cured by using the polyester resins shown in Table 1 and blocked isocyanate as a curing agent. The tackiness of the polyester masking layer was checked with a rolling-ball tester shown in Figure 5.

\section{Results and Discussion}

\subsection{Preparation of the Sliver Paste and Stretchable Polyester PU-Substrate Film}

A HAAKE rheometer was used to measure the rheological properties of the silver paste. As Figure 6(a) shows, the viscosity curves overlapped closely with little hysteresis in the viscosity vs. shear-rate plots. From this result, it was confirmed that the silver-powder mixture was well dispersed in the polyester/ECA binder polymer solution.

From the storage and loss moduli vs. shear stress plots in Figure 6(b), the inversion of loss modulus over storage modulus was observed at a shear stress about $20 \mathrm{~Pa}$. Since the inversion point is usually observed at a stress under 100 $\mathrm{Pa}$ in the silver paste with a poor dispersion of silver particles, the silver paste 


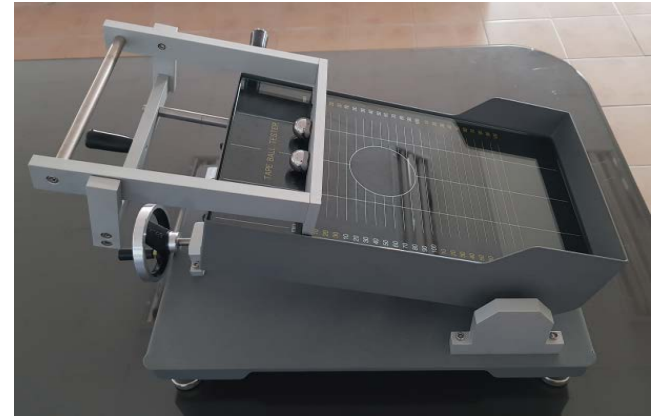

Figure 5. Rolling ball tester for measuring tackiness of the polyester masking layer.

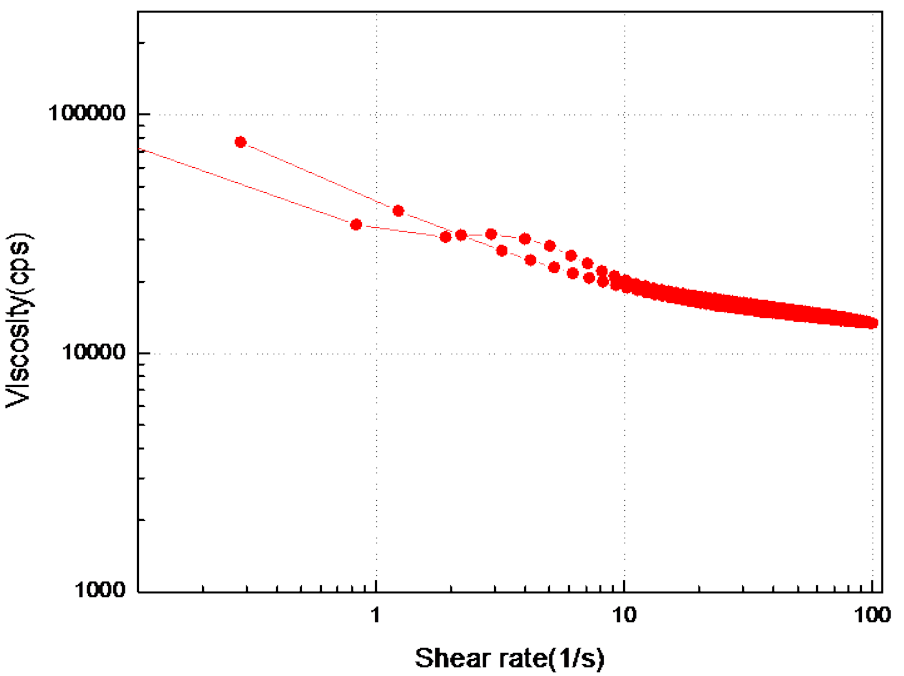

(a)

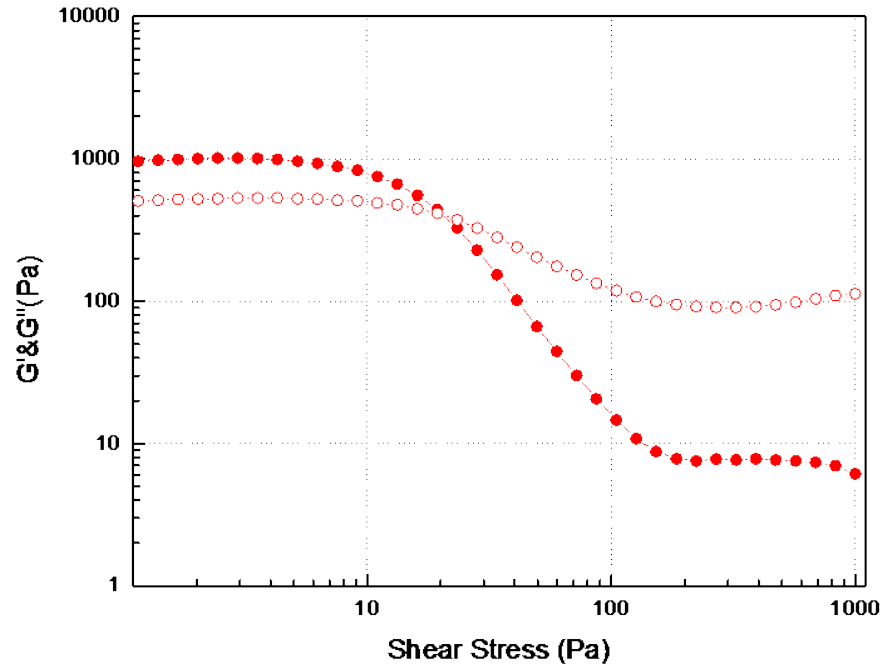

(b)

Figure 6. The (a) viscosity vs. shear-rate plot and (b) storage/loss moduli vs. shear-stress plot.

made by the formulation shown in Table 2 could be assumed to pass smoothly through the openings in the 250-mesh screen plate. 
Elastomeric materials such as PU, PDMS, and EcoFlex were used to fabricate the stretchable strain sensors. We chose CF PU as a substrate material since it has balanced properties in terms of mechanical strength, elasticity, and applicability to screen printing with silver paste. However, the CF PU-substrate films made with Clear Flex 30 PU resins by bar coating followed by thermal curing were too sticky for application of silver paste to screen printing.

From the ball-tack test results in Figure 7 and the crosslinking-reaction mechanism of the polyhydroxy polyester resin and blocked isocyanate in Figure 8, the optimum composition of the masking layer was set to ES-365: ES-360M = 80:20 wt\% polyester resins(renamed as "ES-360S"). This optimum composition may be explained as follows. First, the combination of polyester resins (ES-365 and ES-360M) had an adequate glass-transition temperature of $16^{\circ} \mathrm{C}-17^{\circ} \mathrm{C}$. In the case of polyester resins with a lower $\mathrm{T}_{\mathrm{g}}$ (ES-215 and ES-500), the tackiness of the masking layer is still high for the screen printing of the silver paste. Second, the polyester resin mixture (ES-360S) is suitable for matching the high elasticity of stretchable PU films. The polyester ES-360 has a slightly lower molecular weight and softening temperature, but a higher hydroxyl $(\mathrm{OH})$ value compared with ES-365. Thus, the polyester resin mixture (ES-360S) offers the right level of crosslinking density to the polyester-masking layer while not affecting the good elastomeric properties of the CF PU-substrate film.

\subsection{Screen Printing of Silver Paste and Application to the Strain Sensor}

Although the rheological properties of the silver paste were fine, as shown in Figure 6, it could not be applied directly to the screen printing on the stretchable CF PU films due to high tackiness of the CF PU film. In order to solve the

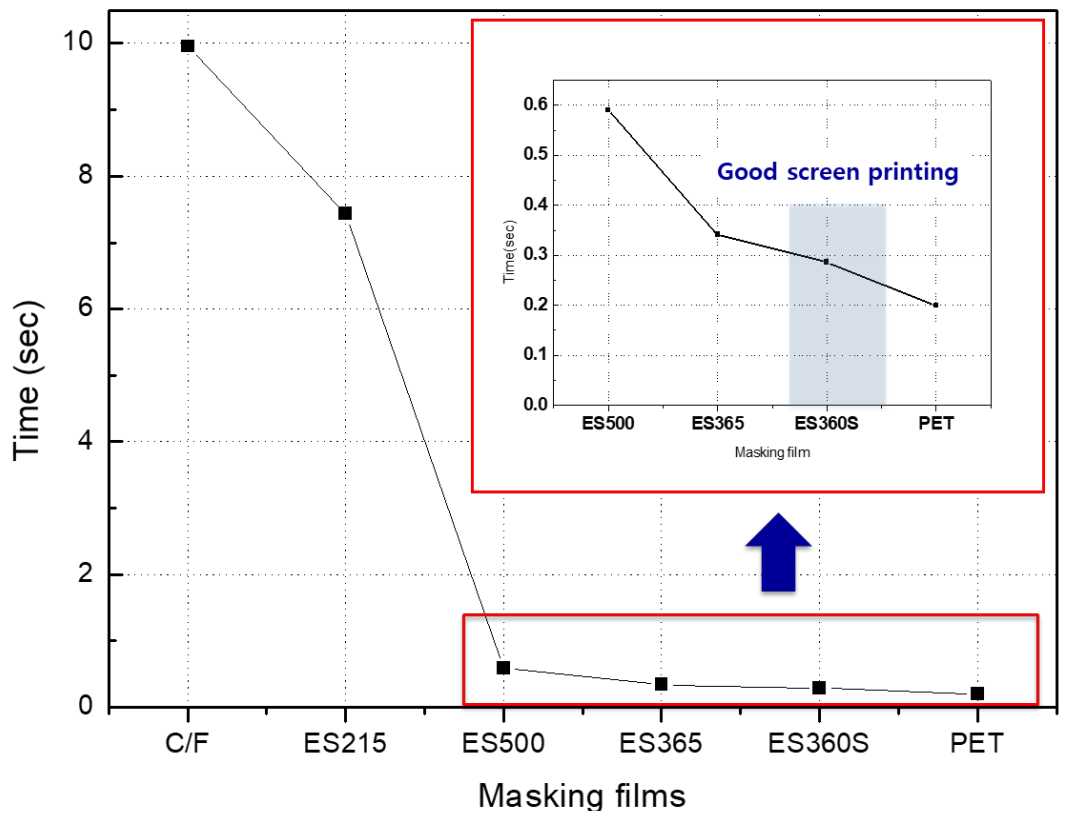

Figure 7. Ball-tack test of polyester-masking layers (CKSI Co., Korea). 

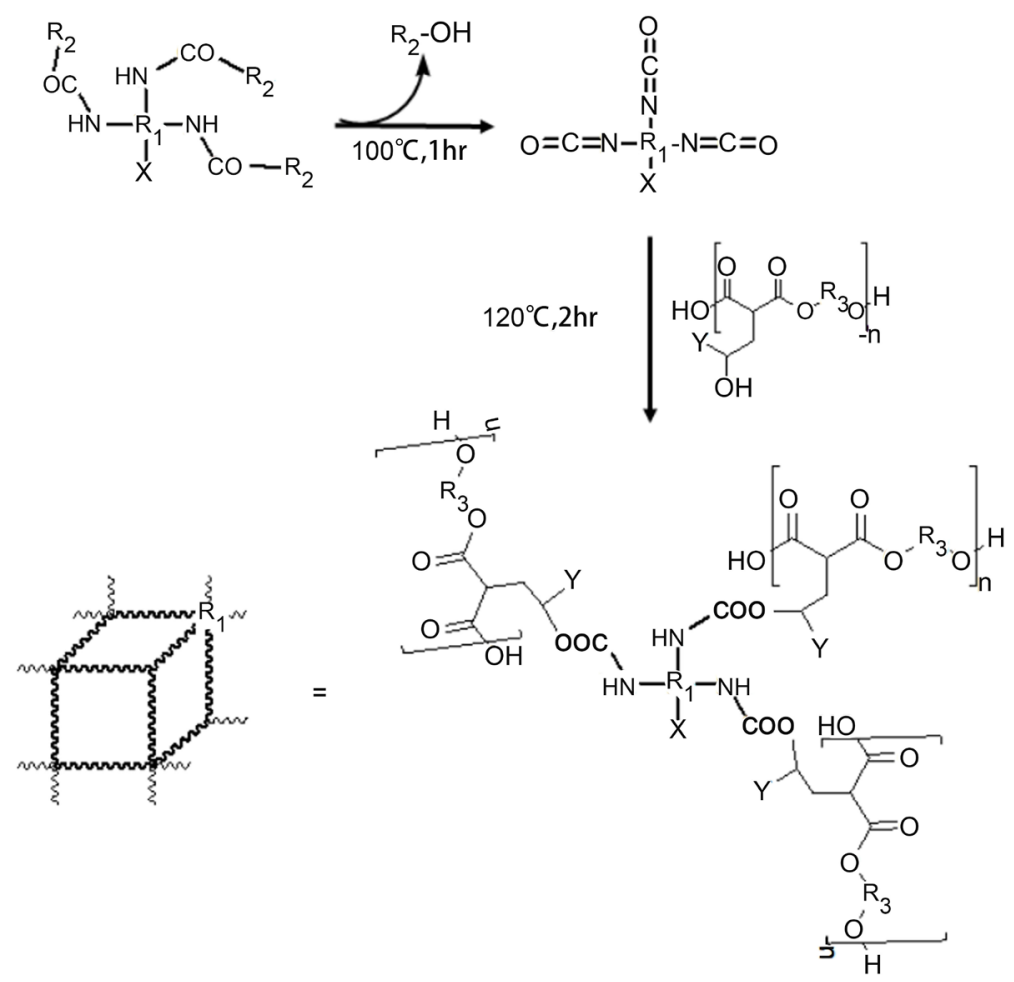

Figure 8. The chemical crosslinking of polyhydroxy polyester resin with a blocked isocyanate curing agent.

problem, both surface modification of the CF PU film and optimization of the Ag paste are required. Of the silver pastes made with the four polyester resins shown in Table 1, that made with polyester ES-215 $\left(\mathrm{T}_{g}=-11^{\circ} \mathrm{C}\right)$ was found to match the elastic property of the CF PU substrate film.

To check why this was the case, TGA analyses of polyester ES-215 itself and a silver-electrode film made with polyester ES-215 resin as a binder polymer were performed as follows. First, the polyester resin ES-215 was dissolved in ECA solvent at 50:50 wt\% and screen printed onto the PET film with a high strength of release coating; then, the solvent was completely dried. The dried polyester ES-215 sheet was peeled off of the PET film, was pulverized, and was subjected to TGA analysis. Second, the silver-electrode film made with polyester ES-215 as a binder polymer was screen printed on PET film dried at $130{ }^{\circ} \mathrm{C}$ for $30 \mathrm{~min}$, peeled off, and then pulverized and subjected to TGA analysis. From the TGA thermograms in Figure 9(a) and Figure 9(b), the two samples exhibited the same $\mathrm{T}_{\mathrm{g}} \mathrm{s}$ of $-11^{\circ} \mathrm{C}$, although the peak height of cured silver-electrode film was smaller than that of polyester ES-215. The same low $\mathrm{Tg}$ values $\left(-11^{\circ} \mathrm{C}\right)$ of the two samples suggested that the binder polymer (polyester ES-215) in the cured silver-electrode film could maintain an elastic property comparable to that of the CF PU substrate film.

The elastic property of the multilayer film consisting of a silver-electrode/polyester masking layer/CF PU-substrate film was also examined by varying the masking layer through four different polyester resins (ES-36S, 


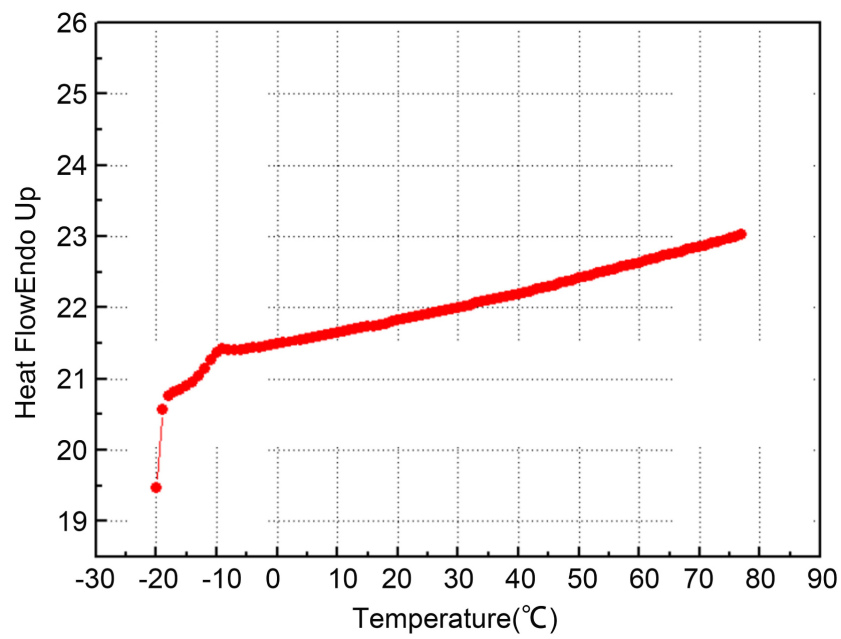

(a)

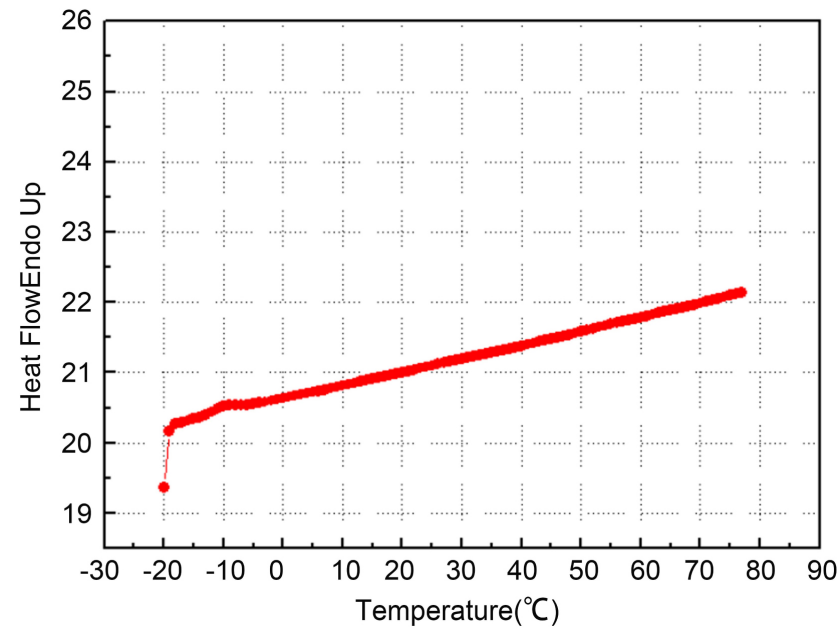

(b)

Figure 9. The TGA thermograms of (a) the polyester ES-215 masking layer and (b) the cured silver-electrode film.

ES-215, ES-500, and Es-360S). The silver-electrode/polyester masking layer/CF PU films were fabricated by repeated coating and thermal curing processes on the PET film with a release layer; the PET film was then peeled off. The silver-electrode patterns made by screen printing of silver paste upon the four different polyester-masking layers were the same as shown in Figure 4. Figure 10 shows the stress vs. strain curves of the multilayer films up to the $40 \%$-strain range.

Of these multilayer films, only that with a polyester ES-360S masking layer exhibited a hysteresis curve without plastic deformation; the others underwent plastic deformation at about $10 \%$ strain. This may be explained by the high hydroxyl $(\mathrm{OH})$ value of polyester ES-360S, as shown in Table 1, potentially enabling matching of the elastomeric property of the CF PU-substrate film by generating chemical crosslinking points through the reaction mechanism shown in Figure 8. 


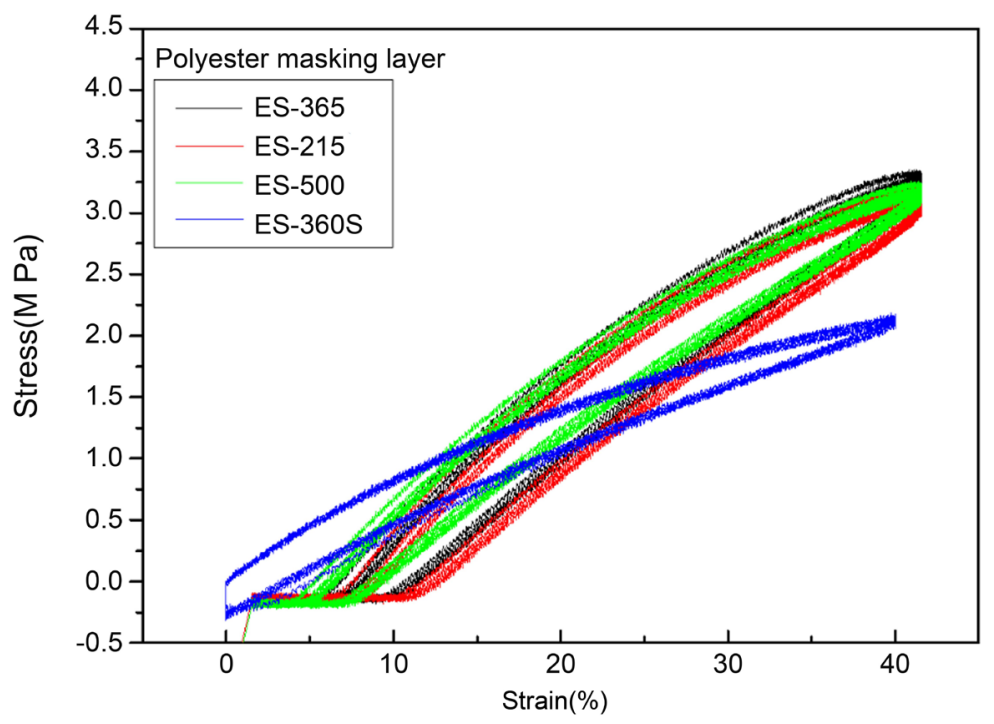

Figure 10. Stress vs strain curves of the silver-electrode/polyester-masking layer/PUsubstrate film.

Having optimized the formulation of the silver paste, the screen printing process was examined using polyester-masking layer/CF PU double-layer films using various different polyester resins (ES-365, ES-215, ES-500, and ES-360S) in the masking layer.

Figure 11 shows the silver-electrode patterns that were screen printed upon different polyester-masking layers. It is apparent that the polyester-masking layer made with polyester resin ES-360S yielded a fine silver-electrode pattern, whereas other polyester resins showed many fringes aligned with the squeezing direction of the screen printer. This was caused by the delayed separation of squeezing from the plate of the screen printer due to the high tackiness of the polyester-masking layer/CF PU-substrate film on which screen printing of the silver paste was conducted. When the PET film itself without a masking layer was used as the substrate, no fringes were observed; however, the width of the silver-electrode pattern was significantly increased compared to that printed on top of polyester ES-360S masking layer/CF PU film. This means that the silver paste becomes slippery when screen printed upon the hard surface of PET film. Thus, it can be noted that applying the polyester masking layer is an important process for exact screen printing of silver electrodes upon highly stretchable CF PU-substrate films.

Figure 12 shows the electrical-resistance $(\Omega)$ vs. strain (\%) curves of the silver-electrode/polyester-masking layer/CF PU-substrate films up to $100 \%$ strain. First, the silver-electrode-multilayer films were stretched from zero to $50 \%$ strain 10 times in order to reduce the residual stress/strain of the silver-electrode-multilayer film, and then the electrical resistances were measured from zero to $100 \%$ strain at several points.

The two sets of strain sensors with silver electrodes (thickness $30 \mu \mathrm{m}$ (Group A) and thickness $60 \mu \mathrm{m}$ (Group B) screen printed on the polyester masking layer 


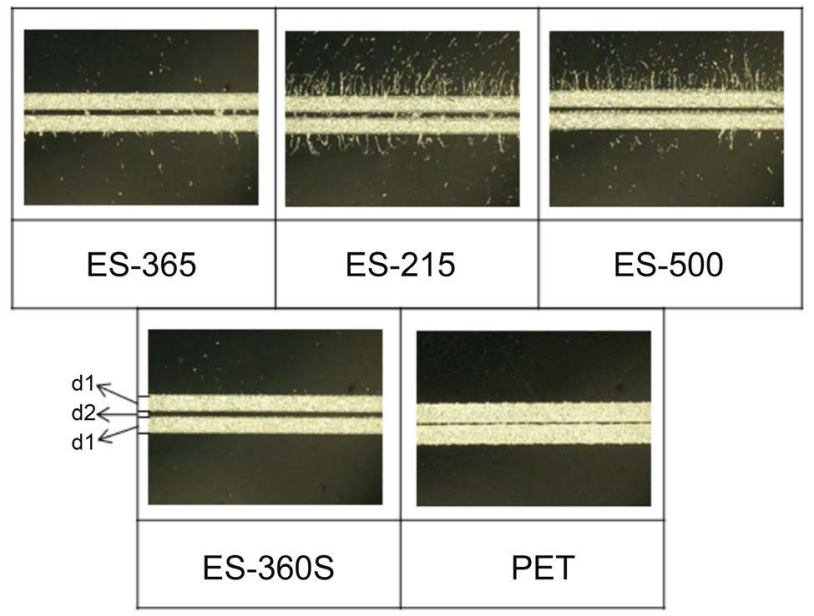

Figure 11. Optical-microscope images (X150) of the screen printed silver-electrode/polyester masking layer/PU-substrate films $\left(d_{1}=200 \mu \mathrm{m}, d_{2}=50 \mu \mathrm{m}\right)$.

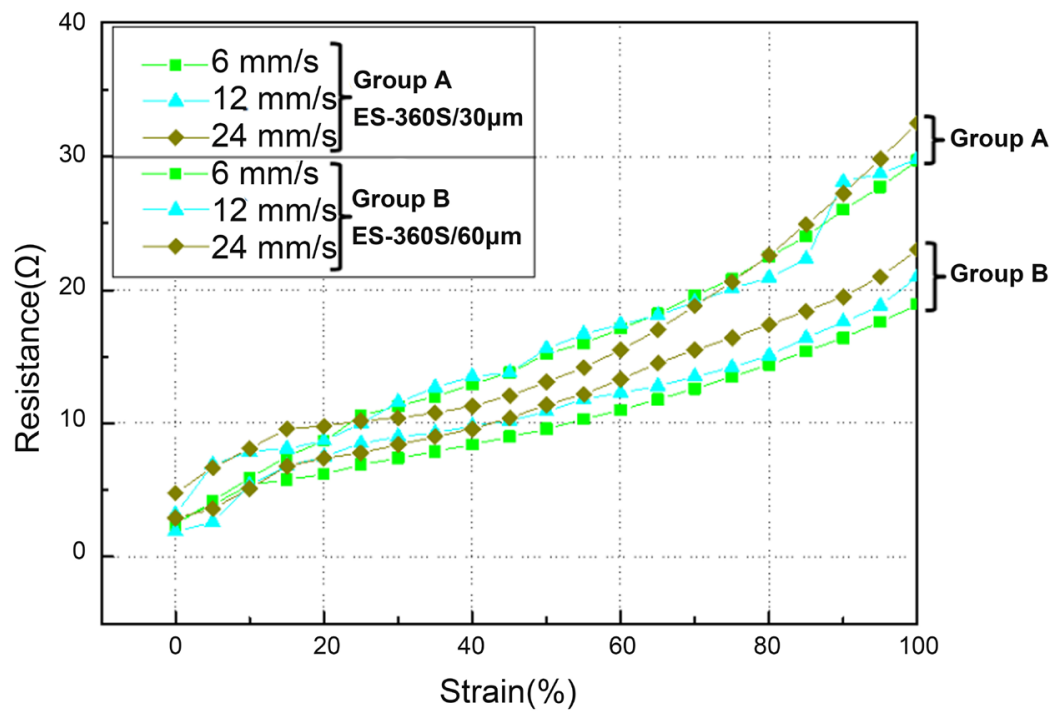

Figure 12. Electrical-resistance vs. strain plots of Ag electrode (Group A: $30 \mu \mathrm{m}, \mathrm{B}: 60$ $\mu \mathrm{m}) /$ polyester masking layer $(3 \mu \mathrm{m}) / \mathrm{PU}(150 \mu \mathrm{m})$-substrate films.

$(3 \mu \mathrm{m}) / \mathrm{CF}$ PU $(150 \mu \mathrm{m})$-substrate films) yielded the following results: 1$)$ both sets of silver-electrode sensors formed by screen printing processes had good linearity up to $100 \%$ strain, considering that the strain range of human hands, arms, and legs falls within $60 \% ; 2$ ) the difference in the electrical resistances between the two sets of strain sensors was less than $7 \Omega$ at a maximum strain of $100 \%$, suggesting that the stability of the strain sensors can be guaranteed regardless of unstable processing conditions during fabrication by the coating and screen printing method; 3 ) the differences in the electrical-resistance values were in the range of 3 to $33 \Omega$, whereas the strain values varied from zero to $100 \%$, potentially giving a fast response by the strain sensors; 4) both sets of strain sensors exhibited good linearity at movement speeds ranging from 6 to $24 \mathrm{~mm} / \mathrm{sec}$. Thinner sensors (Group A) were noted to show smaller changes of electric- 
al-resistance than thicker ones, regardless of movement speed; 5) above all, the stretchable strain sensors could be fabricated using cost-effective materials (silver paste and CF PU-elastomeric films) with a simple sensor structure and screen printing processes that can be applied for sensing a wide range of human body movements.

\section{Conclusion}

In this study, we chose silver paste as sensing material and used a screen printing process to fabricate strain sensors based on the electrical-resistance mechanism. The introduction of a polyester-masking layer on top of the stretchable but tacky CF PU-substrate film made it possible to screen print the silver paste with the exact dimensions required. The strain sensor that incorporated a masking layer exhibited high sensitivity and fast response up to a $100 \%$-strain range, which can be widely used in cost-effective sensors for monitoring human healthcare.

\section{Acknowledgements}

This work was supported by the Technology development Program (S2515668) funded by the Ministry of SMEs and Startups (MSS, Korea).

\section{Conflicts of Interest}

The authors declare no conflicts of interest regarding the publication of this paper.

\section{References}

[1] Webb, R.C., Bonifas, A.P., Behnaz, A., Zhang, Y., Yu, K.J., Cheng, H., Shi, M., Bian, Z., Liu, Z., Kim, Y.S., Yeo, W.Z., Bian, Z., Liu, Y.S., Kim, W.H., Park, J.S., Song, J., Li, Y., Huang, Y., Gorbach, A.M. and Rogers, J.A. (2013) Ultrathin Conformal Devices for Precise and Continuous Thermal Characterization of Human Skin. Nature Materials, 12, 938-944. https://doi.org/10.1038/nmat3755

[2] Mannsfeld, S.C.B., Tee, B.C.-K., Stoltenberg, R.M., Chen, C.V.H.H., Barman, S., Muir, B.V.O., Sokolov, A.N., Reese, C. and Bao, Z. (2010) Highly Sensitive Flexible Pressure Sensors with Microstructured Rubber Dielectric Layers. Nature Materials, 9, 859-864. https://doi.org/10.1038/nmat2834

[3] Lipomi, D.J., Vosgueritchian, M., Tee, B.C.K., Hellstrom, S.L., Lee, J.A., Fox, C.H. and Bao, Z. (2011) Skin-Like Pressure and Strain Sensors Based on Transparent Elastic Films of Carbon Nanotubes. Nature Nanotechnology, 6, 788-792. https://doi.org/10.1038/nnano.2011.184

[4] Cai, L., Song, L., Luan, P.S., Zhang, Q.Z., Zhang, N., Gao, Q.Q., Zhao, D., Zhang, X., Tu, M., Yang, F., Zhou, WB.., Fan, Q.X., Luo, J., Zhou, W.Y., Ajayan, P.M. and Xie, S.S. (2013) Super-Stretchable, Transparent Carbon Nanotube-Based Capacitive Strain Sensors for Human Motion Detection. Scientific Reports, 3, Article No. 3048. https://doi.org/10.1038/srep03048

[5] Cohen, D.J., Mitra, D., Peterson, K. and Maharbiz, M.M. (2012) A Highly Elastic, Capacitive Strain Gauge Based on Percolating Nanotube Networks. Nano Letters, 12, 1821-1825. https://doi.org/10.1021/nl204052z

[6] Kim, K., Hyun, B.G., Jang, J., Cho, E., Park, Y.-G. and Park, J.-U. (2016) Nanoma- 
terial-Based Stretchable and Transparent Electrodes. Journal of Information Display, 17, 131-141. https://doi.org/10.1080/15980316.2016.1240111

[7] Sheng, J., Jeong, H.-J., Han, K.-L., Hong, T. and Park, J.-S. (2017) Review of Recent Advances in Flexible Oxide Semiconductor Thin-Film Transistors. Journal of Information Display, 18, 159-172. https://doi.org/10.1080/15980316.2017.1385544

[8] Trung, T.Q., Tien, N.T., Seol, Y.G. and Lee, N.E. (2012) Transparent and Flexible Organic Field-Effect Transistor for Multi-Modal Sensing. Organic Electronics, 13, 533-540. https://doi.org/10.1016/j.orgel.2011.12.015

[9] Roh, E., Hwang, B.U., Kim, D., Kim, B.Y. and Lee, N.E. (2015) Stretchable, Transparent, Ultrasensitive, and Patchable Strain Sensor for Human-Machine Interfaces Comprising a Nanohybrid of Carbon Nanotubes and Conductive Elastomers. ACS Nano, 9, 6252-6261. https://doi.org/10.1021/acsnano.5b01613

[10] Xiao, X., Yuan, L.Y., Zhong, J.W., Ding, T.P., Liu, Y., Cai, Z.X., Rong, Y.G., Han, H.W., Zhou, J. and Wang, Z. L. (2011) High-Strain Sensors Based on ZnO Nanowire/Polystyrene Hybridized Flexible Films. Advanced Materials, 23, 5440-5444. https://doi.org/10.1002/adma.201103406

[11] Zhang, W.G., Zhu, R., Nguyen, V. and Yang, R. (2014) Highly Sensitive and Flexible Strain Sensors Based on Vertical Zinc oxide Nanowire Arrays. Sensors and Actuators A: Physical, 205, 164-169. https://doi.org/10.1016/j.sna.2013.11.004

[12] Mai, W.J., Liang, Z.W., Zhang, L., Yu, X., Liu, P.Y., Zhu, H.M., Cai, X. and Tan, S.Z. (2012) Strain Sensing Mechanism of the Fabricated ZnO Nanowire-Polymer Composite Strain Sensors. Chemical Physics Letters, 538, 99-101. https://doi.org/10.1016/j.cplett.2012.04.041

[13] Sun, Q., Seung, W., Kim, B.J., Seo, S., Kim, S.W. and Cho, J.H. (2015) Active Matrix Electronic Skin Strain Sensor Based on Piezopotential-Powered Graphene Transistors. Advanced Materials, 27, 3411-3417. https://doi.org/10.1002/adma.201500582

[14] Zhou, J., Gu, Y.D., Fei, P., Mai, W.J., Gao, Y.F., Yang, R.S., Bao, G. and Wang, Z. L. (2008) Flexible Piezotronic Strain Sensor. Nano Letters, 8, 3035-3040. https://doi.org/10.1021/nl802367t

[15] Yao, S.S. and Zhu, Y. (2014) Wearable Multifunctional Sensors Using Printed Stretchable Conductors Made of Silver Nanowires. Nanoscale, 6, 2345-2352. https://doi.org/10.1039/c3nr05496a 